\title{
A behavioral field approach to general activity: Sex differences and food deprivation in the rat
}

\author{
PAUL T. P. WONG \\ Trent University, Peterborough, Ontario, Canada
}

\begin{abstract}
Male and female rats under different levels of food deprivation were observed in an enclosed chamber equipped with several manipulanda. Dependent variables included: time spent in immobility, grooming, exploration, and different types of manipulation (e.g., sanddigging); sequential dependencies between different kinds of behaviors; and ultrasonic activity counts. Results showed that deprivation generally increased exploration and manipulation and decreased immobility. Deprivation also increased the variability of bout lengths but had little effect on sequential dependencies or activity counts. These results provide evidence for the response-selection function rather than the energizing function of food deprivation. Various sex-related factors appearing in the data were also described. The present findings of sex differences and deprivation effects were presented as examples of state-generated behavioral fields.
\end{abstract}

General activity refers to the spontaneous activity of an animal in the absence of any identifiable eliciting or reinforcing stimulus. General activity is typically measured in running wheels, stabilimeter cages and open fields (Baumeister, Hawkins, \& Cromwell, 1964; Gross, 1968; Valle, 1975). With a few qualifications, previous studies have shown that at least in rats food deprivation increases general activity and that females are more active than males (Bolles, 1967; Hughes, 1968; Valle \& Bols, 1976).

The present experiment employed a behavioral field approach to determine the effects of food deprivation and sex on general activity. A description of the behavioral field approach has been given elsewhere (Wong, 1977). In the present context, this approach combined direct observation with automatic, multiple recording to measure different components of general activity as well as their interrelations.

Four mutually exclusive categories of behaviormanipulation, exploration, grooming, and immobility - were observed and recorded by operating a manual keyboard. These categories of behavior represent different degrees of vigor and interaction with the environment. Manipulation consisted of direct manipulation of any of the six manipulanda (foodcup panel, bar, metal blades, chain, drinking spout, and a sanddigging platform) which provided automatic recording of foodcup-panel nosing, barpressing, blade-biting, chain-pulling, drinking, and sand-

Supported by NRC Grants A8635 and A0701. The author gratefully acknowledges the assistance of Philip Moore, Nick White, Wanda Grife, and Janet Olds, especially their patience in processing Esterline Angus charts. Requests for reprints should be addressed to Paul T. P. Wong, Department of Psychology, Trent University, Peterborough, Ontario, K9J 7B8, Canada. digging. Exploration consisted of activities that increased the rat's contact with the environment, i.e., locomotion, rearing, sniffing, hopping, and gnawing at grid. Grooming included all activities directed to various parts of the body, i.e., face-washing, scratching, tail-biting, and licking (belly, sides, or legs). Immobility consisted of crouching, lying, sitting, or standing still, with at least three feet on the floor and the body completely motionless, except for occasional small and slow lateral movements of the head.

Since occurrences of the four major categories of behavior and the six different types of manipulatory behavior were recorded on an Esterline Angus chart, several dependent variables could be derived from the data in addition to time spent in each of these behaviors. A switch from one behavior to another constituted a transition. Frequency of transition from one behavior to another provided an index of sequential dependencies between different bahaviors (Bolles, 1960). Different transitions and different bout lengths were summed to provide an index of the variability of behavioral fields.

An ultrasonic activity recording device as described by Bolles and Sanders (1969) was used to measure the vigor of general activity. A behavioral checklist, consisting of all the components previously listed under each of the four broad categories of behavior, provided a measure of the "complexity" of the behavioral fields.

The present behavioral field approach to general activity thus represents a major extension of previous research with respect to both the range of activity observed and the diversity of measures obtained. 


\section{METHOD}

\section{Subjects}

The subjects were 15 male and 15 female light-hooded rats of Walker-Walker stock bred in the vivarium of Trent University. They were approximately 100 days old and had been handled for several days prior to experimentation. They were housed in single cages in a room with constant illumination.

\begin{abstract}
Apparatus
The testing chamber was enclosed in a sound-attenuated box illuminated by a $5-\mathrm{W}$ houselight and equipped with an exhaust fan and a $17 \times 11 \mathrm{~cm}$ one-way mirror. The chamber was made of Plexiglas with inside dimensions measuring $20.5 \mathrm{~cm}$ wide $x$ $23 \mathrm{~cm}$ long $\times 19.5 \mathrm{~cm}$ high. The foodcup had a $4.5 \times 4.5 \mathrm{~cm}$ opening covered by a free-hanging Plexiglas panel and was $3 \mathrm{~cm}$ deep. When the rat nosed its way into the foodcup and pushed the panel against the back wall, a microswitch was activated. The foodcup was unbaited and thoroughly washed to remove all traces of food. A steel bar, measuring $5.3 \mathrm{~cm}$ wide $\times 1.3 \mathrm{~cm}$ deep $\times 1.2 \mathrm{~cm}$ thick, protruded $6 \mathrm{~cm}$ above the grid floor. The front side of the bar was extended to form a pair of metal blades, each measuring $4.1 \mathrm{~cm}$ wide $\times 1.2 \mathrm{~cm}$ deep, and $2 \mathrm{~mm}$ apart. The upper blade was fixed, but the lower blade could be pressed against the upper one by rats only through biting both blades. A minimum of $125 \pm 5 \mathrm{~g}$ of force, as measured by an Ohaus spring balance, was needed to close the two blades and activate a microswitch. Thus, pressing the bar downward provided a barpressing measure, while pressing the lower blade upward provided a measure of blade-biting behavior. The sanddigging apparatus similar to the one described in Wong, Roach, and Osborne (1975), had a platform, measuring $7 \mathrm{~cm}$ wide $\times$ $6 \mathrm{~cm}$ deep, and $3 \mathrm{~cm}$ above the grid floor. A metal chain, $13 \mathrm{~cm}$ long, was hung from the lid of the testing chamber, with the lower end $6.5 \mathrm{~cm}$ above the grid floor. A drinking bottle was attached to the testing chamber, opposite to the sand-digging assembly. Altogether, the chamber contained six manipulanda which, together with a manual keyboard, were connected to an Esterline Angus chart recorder. An ultrasonic activity recording device as described by Bolles and Sanders (1969) was calibrated such that it was not activated when the rat exhibited immobility as previously described; the recording device would start counting whenever the rat was engaged in grooming, exploration, and manipulation.
\end{abstract}

\section{Procedure}

Deprivation. On the first day of testing, all subjects had been on ad-lib feeding. Following the first testing session, food was removed from subjects in two deprivation conditions, but not from no-deprivation (ND) controls. Thereafter, high-deprivation (HD) subjects were given $7 \mathrm{~g}$ per day until each subject was reduced to $70 \%$ of predeprivation body weights; low deprivation (LD) subjects were given $10 \mathrm{~g}$ per day until each subject was reduced to $80 \%$ of its free-feeding body weight. Once a subject had lost the predetermined amount of body weight (typically within 10 days), its body weight was monitored closely and its daily ration adjusted to maintain the appropriate body weight at the same level throughout the experiment.

Testing. There were 16 testing days. Males and females were run as two separate squads to minimize the confounding of sex odors. The subjects were run 5 days per week and were not tested during weekends. On each testing day, the rats were placed inside the testing chamber one at a time for $10 \mathrm{~min}$ of continuous recording and direct observation through a one-way mirror. Testing sessions were limited to $10 \mathrm{~min}$ because of the limited holding capacity of the sand funnel. The running order within each squad was randomized daily. HD and LD subjects were given their daily rations of food in their home cages at least $1 / 2 \mathrm{~h}$ after all subjects within the same squad had com- pleted their testing. The average interval between testing and feeding for the deprived animals was approximately $2 \mathrm{~h}$.

Recordings. The Esterline Angus chart speed was set at $6 \mathrm{in} . / \mathrm{min}$. Five graduations at $1 / 10$-in. intervals provided 1 -sec markers. Occurrences of the six types of manipulatory behavior and the four mutually exclusive categories of behavior were recorded continually on the chart in each 10 -min session. The duration of each recorded behavioral event was calculated to the nearest second by counting the number of $1-\mathrm{sec}$ intervals. Automatic recording of the six types of manipulatory behavior was precise enough to permit the measurement of run or bout length. A run consisted of a single occurrence longer than $1 \mathrm{sec}$ in duration or several occurrences of the same type of manipulatory behavior no more than $3 \mathrm{sec}$ apart, and the length of run was then determined by counting the number of $1-\mathrm{sec}$ intervals of this cluster of occurrences. The number of different lengths of runs of all manipulatory behaviors for each rat in each session was calculated. For example, if a rat had two bouts of sand-digging with different run lengths (e.g., 2 and $4 \mathrm{sec}$ ) and one bout of drinking in a particular session (e.g., $7 \mathrm{sec}$ ), then the number of different lengths of runs for this rat was three. Transition matrices involving only the manipulatory behaviors as well as involving both the manipulatory behaviors and other categories of behavior were extracted from the Esterline chart for each rat in each session. A transition was defined by a change from one type of manipulatory behavior to another type, or from one category of behavior to another category. Both the frequency of each kind of transition as well as the number of different transitions for each rat in each session were recorded. For example, a rat may have three sand-digging-to-grooming transitions, two groomingto-sand-digging transitions, and one grooming-to-drinking transition; in this case, the number of different transitions was three. Different lengths of runs combined with different transitions provided an index of the variability of behavioral fields.

\section{RESULTS}

\section{Time Spent in Different Behaviors}

Figure 1 shows that all four categories of behavior were sensitive to weight loss, and the systematic effect of deprivation was more clearcut in males. In manipulation, exploration, and immobility, the effect of deprivation increased with days of deprivation, resulting in a significant Deprivation by Days interaction $[\mathrm{Fs}(30,360)=2.51,2.26$, and 1.87 , re-

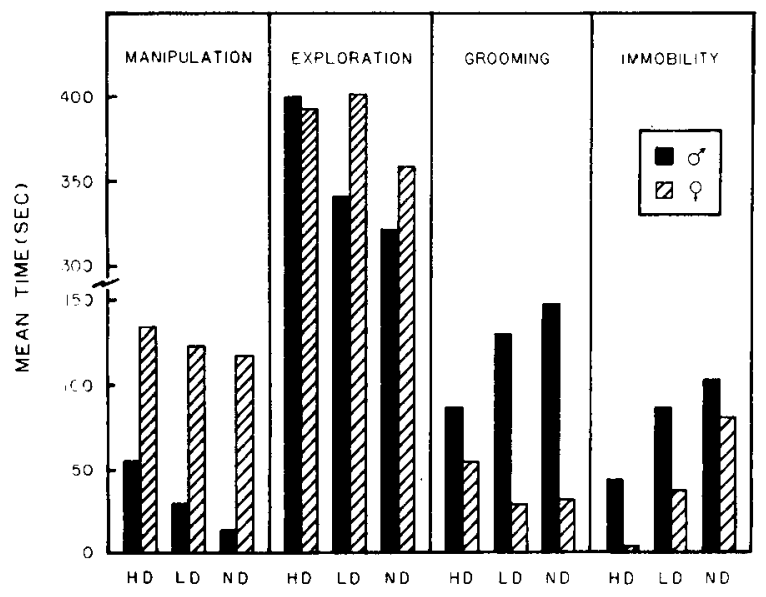

Figure 1. Mean time spent in different categories of behavior across all testing days. 
spectively, $\mathrm{p}<.01$ in all cases]. The main effect of deprivation in immobility was also significant $[F(2,24)$ $=4.54, \mathrm{p}<.05]$. The present finding that deprivation increased manipulatory and exploratory behavior but decreased immobility is quite consistent with that observed in golden hamsters (Shettleworth, 1975). With respect to grooming, deprivation interacted with sex $[F(2,24)=5.67, p<.01]$ such that, as deprivation level increased, grooming decreased in males but increased in females (at least between HD and LD females). The main effect of sex in grooming was also significant $[\mathrm{F}(1,24)=94.70, \mathrm{p}<.01]$, with males spending more time in grooming; this is consistent with Bolles' (1960) observation that male rats spent a considerable amount of time grooming.

Sex differences were obtained in other behavioral categories as well. Females spent more time in manipulatory behavior, but mainly during the last few sessions, resulting in a significant Sex by Days interaction $[F(15,360)=2.47, p<.01]$. During the early sessions of testing, both males and females exhibited a very low level of manipulation due to a very strong exploratory tendency in females and a high level of exploration and immobility in males.

In exploration and immobility, sex differences were most pronounced in the initial sessions and gradually decreased, as evident in a significant Sex by Days interaction $[\mathrm{Fs}(15,360)=3.82,1.68, \mathrm{p}<.01, \mathrm{p}=$ .05 , respectively]. During the first three sessions, mean times spent by males and females were 337 vs. $477 \mathrm{sec}$ in exploration, and $105 \mathrm{vs}$. $25 \mathrm{sec}$ in immobility. These differences diminished with additional sessions of testing. In exploration, males even surpassed females slightly (363 vs. 321 ) in the last session. A similar cross-over effect has recently been reported by Russell (1977).

Separate analyses of variance on time spent in the six different types of manipulatory behavior revealed significant group differences only in sand-digging and foodcup-nosing, as shown in Figure 2. Occurrences of chain-pulling, drinking, barpressing, and blade-biting were rare for all subjects. The main effect of deprivation in sand-digging and nosing was significant $[\mathrm{Fs}(1,24)=4.21,3.46$ respectively, $\mathrm{p}<$ .05 in both cases]. Orthogonal comparisons showed that these variances were largely due to the differences between deprived rats (HD and $L D$ ) and ND controls $[\operatorname{Fs}(1,24)=6.99,5.07, p<.05$ in both cases]. The effect of deprivation in sand-digging and foodcup-nosing increased with days of deprivation, resulting in a significant Deprivation by Days interaction $[\mathrm{Fs}(30,360)=2.06,2.38, \mathrm{p}<.01$ in both cases]. The main effect of sex was also significant in both sand-digging and foodcup-nosing $[\mathrm{Fs}(1,24)$ $=14.59,17.59, \mathrm{p}<.01$ in both cases] .

\section{Activity Counts}

Activity counts, as a measure of response vigor,

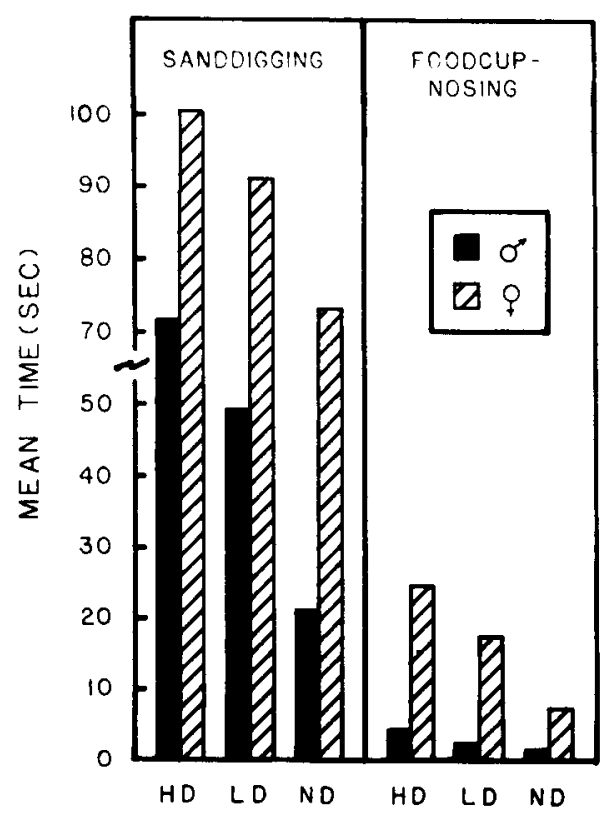

Figure 2. Mean time spent in sand-digging and foodcup-panel nosing across all testing days.

were provided by an ultrasonic device. Unfortunately, the first 2 days of activity counts of males and the entire activity counts of female HD and LD subjects were lost. ${ }^{1}$ The mean activity counts for the male ND, LD and HD groups were 1,390.0, 1,474.4, and $1,516.5$, respectively. Thus, activity counts seemed to be positively related to the level of deprivation in males, but neither the main effect of deprivation $(F<1.0)$ nor the Deprivation by Days interaction $[\mathrm{F}(26,156)=1.05, \mathrm{p}=.41]$ was significant. The female ND group had a significantly higher activity count than its male counterpart, $1,778.2$ vs. $1,390.0 \quad[\mathrm{~F}(1,8)=14.28, \quad \mathrm{p}<.01]$. However, like exploration, this difference occurred mainly during the initial sessions of training, and diminished gradually, resulting in a significant Sex by Day interaction $[F(13,104)=3.94, p<.01]$. During the first three sessions, mean activity counts for male and female ND groups were 1,700 vs. 2,100 . In the last session, activity counts for male and female ND groups were 1,095 vs. 802 , with males surpassing females.

\section{Sequential Dependencies Between Different Behaviors}

The organizational or structural aspects of behavioral fields can be expressed in terms of sequential dependencies between different behaviors. In the present study, first-order sequential dependencies were calculated by averaging over the entire 16 sessions the frequencies of one behavior being immediately preceded and followed by a different behavior. Figure 3 shows the first-order sequential dependencies between foodcup-nosing (FC), sand-digging (SD), chain-pulling (CP), and drinking (DK). Barpressing 


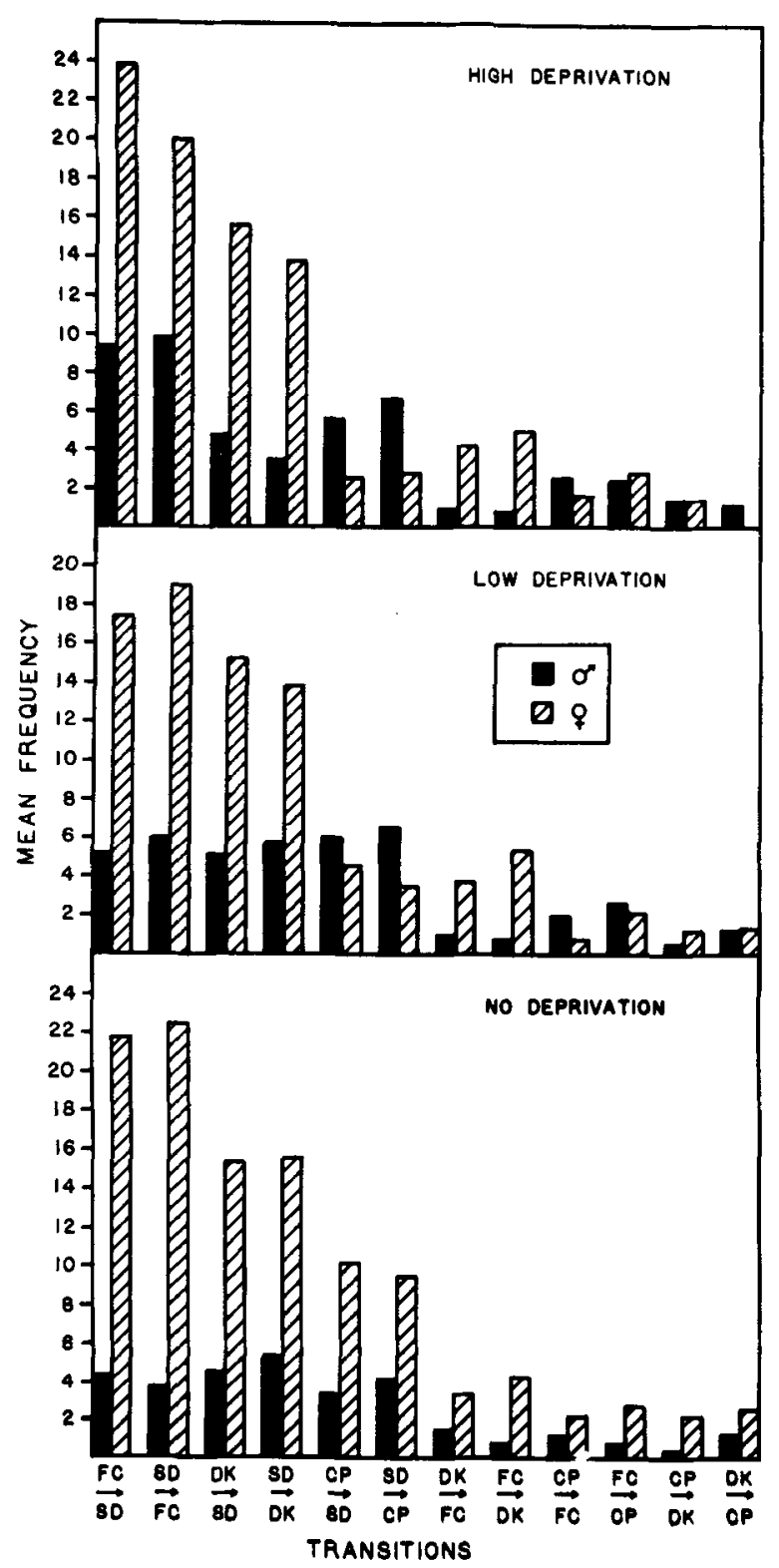

Figure 3. First-order sequential dependencies between four types of manipulatory behavior: Foodcup-nosing (FC), sand-digging (SD), chain-pulling (CP), and drinking (DK).

and blade-biting were excluded because of their extremely rare occurrence. Several observations may be abstracted from the graph. First, food deprivation had little or no effect on sequential dependencies of different manipulatory behaviors. There was only some slight indication that transitions between FC and SD increased with deprivation in males. Secondly, sex differences in sequential dependencies were quite pronounced. In general, transitions were more frequent but less evenly distributed in females. Thirdly, in some cases, deprivation and sex interacted. For example, deprivation increased transitions between SD and CP in males, but decreased these transitions in females. Fourthly, transitions between any two manipulatory behaviors were symmetric or bidirectional in most cases. That is, the frequency of a response $\mathrm{A}$ followed by a response $\mathrm{B}$ was about the same as B followed by $A$.

Sequential dependencies involving the same four manipulatory behaviors plus immobility (IM) and grooming (GM) are depicted in Figure 4. Exploration was excluded because the spatial arrangement of manipulanda was such that the transition from certain manipulatory behaviors (e.g., SD) to another (e.g., FC) necessarily involved locomotion, which was treated as a subset of exploratory behavior in the present study; thus, transitions involving exploration were inflated. Figure 4 shows that once again the effect of deprivation was minimal; there appeared to be an inverse relationship between SD-IM transitions and deprivation in females only. Sex differences, on the other hand, were much more clearcut. In males, GM-IM transitions were more than three times higher than those in females, but the linkage between SD and IM was much stronger in females than in males, especially in the ND condition. Bolles (1960, p. 308) reported that "grooming sequences tend to disproportionately follow sequences of eating and especially drinking, and to be followed by sleeping and miscellaneous activity." In the absence of food, as in the present experiment, grooming was most frequently preceded by immobility.

The summation of the frequencies of different kinds of behavioral transitions by each rat in each session yielded a score of total number of transitions. This measure simply indicated now often the rat switched from one behavior to another. Mean numbers of total transitions averaged over the entire 16 sessions for different groups are plotted in the left panel of Figure 5. Analysis of variance revealed a significant sex main effect $[F(1,24)=8.12, p<.01]$, but this sex difference occurred mainly in $L D$ and ND conditions as supported by a significant Sex by Deprivation interaction $[F(1,24)=3.32, p<.05]$. The higher total number of transitions of $L D$ and ND males was largely due to a disproportionately high frequency of transitions between immobility and grooming, as shown in Figure 4.

\section{Variability and Complexity of Behavioral Fields}

Different transitions and different lengths of runs are presented in the middle two panels of Figure 5. The graph shows that females had more different transitions and more different lengths of runs than males, as supported by a significant main effect of $\operatorname{sex}[\operatorname{Fs}(1,24)=23.27,39.43, p<.01$ in both cases]. In other words, behavioral transitions in females were more varied and the bout lengths of their manipulatory behaviors were also more varied. When these two measures were summed, as shown 


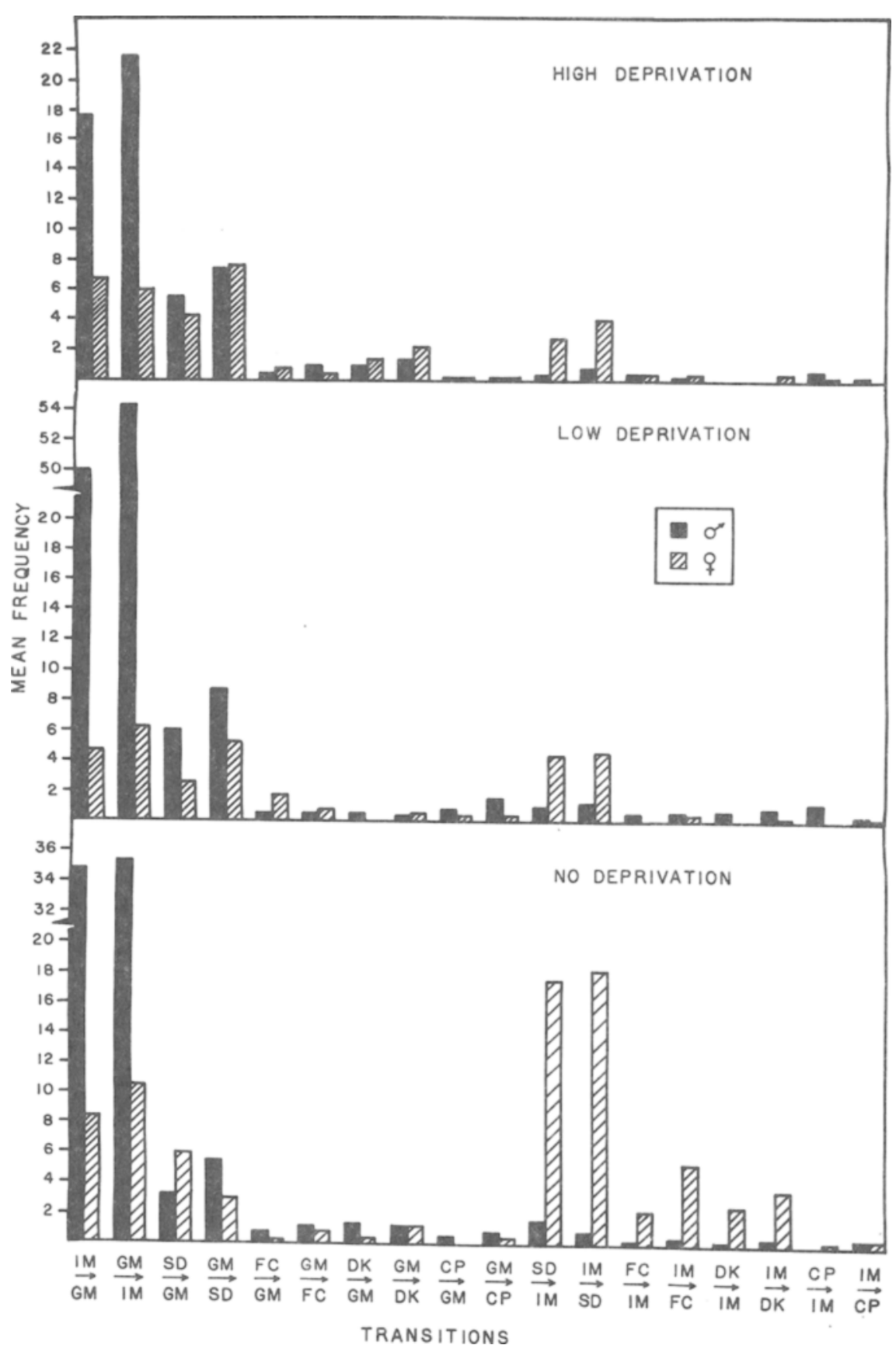

Figure 4. First-order sequential dependencies between immobility (IM), grooming (GM), and the four types of manipulatory hehavior.

in the right panel of Figure 5, they provided an overall index of variability of behavioral fields. Analysis of variance confirmed that females had a significantly higher overall variability score $[F(1,24)=50.90$, $\mathrm{p}<.01]$.

Deprivation had a more limited effect on behavioral variability than sex. A significant main effect of deprivation was obtained only in different bout lengths $[F(2,24)=4.46, p<.05]$, and this effect was limited to the difference between deprived (HD and LD) and ND subjects $[F(1,24)=5.16$, $p<.05]$. Neither the deprivation main effect nor the Deprivation by Sex interaction was significant in different transitions and the overall variability score.

The complexity dimension failed to differentiate between groups, with all group means clustering around a score of 9 , ranging from an 8.73 to 9.74. Thus, the number of different response topo- 


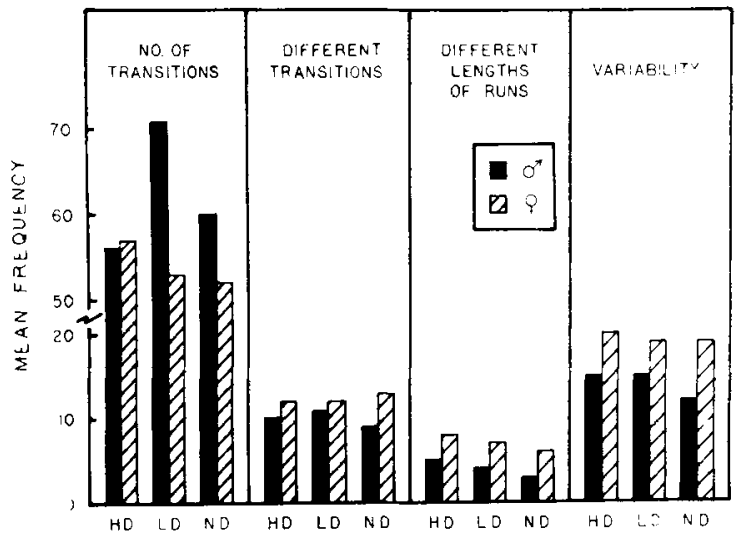

Figure 5. Mean frequency of total number of transitions (left panel), different types of transitions and different lengths of runs (middle two panels), and the overall variability measure (right panel) across all testing days.

graphies comprising the behavioral field was not affected by food deprivation or sex. In all groups, walking, sniffing, rearing, face-washing, licking, scratching, sitting down, and sand-digging occurred most consistently, while blade-biting and hopping occurred most rarely.

\section{DISCUSSION}

\section{Food Deprivation}

The present behavioral field approach to general activity has demonstrated that the effect of food deprivation is not limited to wheel-running and exploration as hitherto reported in the literature. In the present study, all four broad categories of behavior were sensitive to weight loss, but not always in the direction of heightened activity. For example, deprivation actually decreased grooming in males. The effect of deprivation on manipulatory behaviors was obtained mainly in sand-digging and foodcupnosing. It is difficult to conclude that these findings support Hull's (1943) notion that food deprivation which contributes to generalized drive (D) should energize whatever behavior the animal is engaged in. Alternatively, the present findings may be interpreted as supporting a response-selection function of drive. It may be hypothesized that food deprivation only selectively lowers the response evocation threshold of behaviors that are ecologically related to food-seeking or food-gathering. Both Berlyne (1966) and Hull (1943) have pointed out that exploration increased the likelihood of finding food. Sand-digging and nosing responses can be considered as components of species-specific burrowing behavior, which is so common in rodents (e.g., Deutsch, 1960; King \& Weisman, 1964) and which may also be ecologically related to food-seeking and foodgathering. Bolles (1967, p. 289) stated that "we can no longer accept general activity as a direct measure of drive strength." In light of the present findings, it may be argued that specific components of general activity, such as sand-digging, may be a direct measure of deprivation level or drive strength.

The present findings of deprivation effects are not readily attributable to associative factors of food-expectancy for the following reasons. First, none of the subjects had any prior experience of food deprivation when testing began. Secondly, the deprived rats were never fed in the testing chamber, and feeding took place in the home cage, usually $2 \mathrm{~h}$ after testing. Thirdly, if the present deprivation effects were due to food expectancy or incentive, one would expect exploration and manipulation to increase towards the end of each $10-\mathrm{min}$ stimulus. However, when the data were broken down into 1-min intervals, it was found that for most subjects these two categories of behavior decreased while immobility increased in the last $2 \mathrm{~min}$ of each session. Therefore, it seems most appropriate to attribute the present deprivation effects to the in ternal, organismic state of food deprivation.

If one considers exploration and manipulation as reflecting more vigorous activities than grooming and immobility, one would conclude that deprived rats were clearly more active than ND subjects. However, in terms of ultrasonic activity counts, deprivation seemed to have little effect on the level of activity. It has been reported that ultrasonic counts increased with heightened level of activity (Bolles \& Sanders, 1969); for example, rats produced more ultrasonic counts moving around than grooming or sitting still. In the present study, female ND subjects who were more active than male counterparts in terms of time spent in exploration and manipulation also had higher ultrasonic counts. Thus, the ultrasonic recording device does seem to provide a valid measure of general activity. The reason that this device failed to significantly differentiate between deprivation levels might be because its sensitivity level was set too high. In the present study, the recording device was so calibrated that it would cease producing counts only when the animal was completely motionless. It is likely that grooming may produce the same rate of counts as sand-digging and nosing, because both these behaviors, like grooming, do not involve locomotion. Should the sensitivity level be lowered so that only gross bodily movements such as locomotion or rearing could produce counts, a significant deprivation effect might be obtained.

The present study also shows that deprivation had no appreciable effects on sequential dependencies, total number of transitions, different transitions, and the combined variability score. Only different lengths of runs of manipulatory behaviors were affected by deprivation, but this difference might simply be due 
to the fact that deprivation also increased the bout frequency of sand-digging and nosing. When the occurrence of a behavior increases, the variability of its bout lengths should increase as well.

\section{Sex Differences}

Sex differences in general activity as measured by the present behavioral field approach were more complex and pervasive than mere differences in the level of activity. In manipulatory behaviors (i.e., sand-digging and nosing), sex differences occurred mainly in the later sessions of testing; in exploration, sex differences occurred mainly in the early sessions. Females not only had higher activity counts, but also exhibited greater behavioral variability as measured by different transitions and different bout lengths. Further, the pattern of sequential dependencies in females was qualitatively different from that of males. For example, given the occurrence of immobility, the prediction of what behavior is most likely to follow will depend on the sex of the rat being observed. In males, immobility is most likely to be followed by grooming; in females, it is more likely to be followed by sand-digging, at least in the no-deprivation condition. These differences in sequential dependencies reinforce the present author's contention that behavior has structure, and that organismic states, whether induced by hormone or drug, may affect both specific components of general activity and their sequential structure. For example, Norton (1973) has demonstrated that amphetamine not only increases the frequency and duration of various acts, but also alters their temporal patterns.

Sex differences in exploratory behaviors have been subjected to various interpretations (e.g., Archer, 1975). For example, the higher ambulation score by females has been attributed to both lower and higher "emotionality" of females (Archer, 1973; Gray, 1971; Halliday, 1968). Further, Archer (1975) has suggested that sex differences may be simply due to different forms of fear-responding, with immobility being the predominant fear response in males and active escape/avoidance being dominant in females. However, the present finding of a crossover effect in the level of exploration suggests that sex differences may be best accounted for in terms of different responsiveness to novelty. In other words, given the same novel environment, males' responsiveness may be stronger than that of females. If one also assumes that moderate responsiveness to novelty results in exploration while a strong responsiveness to novelty results in immobility, then the present cross-over effect can be readily predicted. During the initial sessions of testing, due to their strong responsiveness to novelty, males should have had a higher probability of immobility response but a lower probability of exploration than females, and this was exactly what was observed. With repeated trials, responsiveness should decrease due to habituation. In females, the peak level of exploration should occur during the initial sessions, to be followed by a decremental trend throughout testing. In males, the peak level of exploration occurred only when the immobility reaction to novelty had habituated; thus exploration should first increase and then decrease. These different trends of exploratory behavior in males and females were indeed obtained, as evident in a significant Sex by Days interaction cross-over. Therefore, sex differences in the present study can be explained by assuming different initial responsiveness to novelty without the additional assumption of different rates of habituation.

Sex differences in manipulatory behavior occurred primarily in the later sessions, because reactions to novelty (exploration and immobility) were stronger than manipulation in the early sessions. The fact that sex differences were limited to sand-digging and nosing suggests that these differences were not simply due to higher motor activities in females. It is interesting to note that sex differences parallel deprivation differences. Just as food-deprived rats spent more time in sand-digging and nosing, female rats also spent more time in these two types of behavior. Such resemblance implies a greater extent of deprivation in female rats. It is likely that, in females, sand-digging and nosing (as components of burrowing) were motivated by both mate-seeking and foodseeking. That general activity is higher at estrus than at diestrus (Birke \& Archer 1975; Finger, 1969; Wang, 1923) does provide some evidence for the mate-seeking hypothesis (Russell, 1977). Females' higher variability scores (as measured by different transitions and different bout lengths) and higher activity counts may also be related to estrus cycles. In other words, food deprivation and sexual deprivation in female rats (especially when they are in heat) may have an additive effect on behavior.

\section{Conclusions}

The present experiment has demonstrated that a behavioral field is more than the sum of its various behavioral components; that behavioral fields have sequential structures (i.e., sequential dependencies) as well as other properties that transcend individual behavioral components (i.e., complexity, variability, and activation). The behavioral field approach simply attempts to measure both structural properties and individual behavioral components. The usefulness of this approach has been demonstrated in identifying hitherto unnoticed aspects of sex differences and deprivation effects. Inasmuch as these differences are not readily accountable in terms of identifiable eliciting stimuli or reinforcing consequences, they 
may be attributable to organismic states. In other words, the present effects of sex and deprivation on general activity may be conceptualized as different behavioral fields generated by different internal states.

One may argue that state-generated behavioral fields represent the most important and fundamental category of behavior, which constitutes the provenance of both respondents and operants (cf. Segal, 1972). Unfortunately, due to the prevailing tendency to externalize the source of motivation in the past two decades, learning psychologists have concentrated on the elicitation and consequence operation as employed in classical and operant conditioning, respectively, and have largely ignored the deprivation-stimulation-injection operations as antecedents of organismic states (cf. Brown, 1961). In recent years, there are signs of renewed interest in internal sources of motivation. For example, Bindra (1969) has emphasized the importance of central motive states in learning, and conceptualized central motive states as "generated by the neural representations of organismic state (deprivation) and incentive object"' (p. 12). It is very likely that conditionability of any response in both classical and operant paradigms may depend on its relationship with other response components in a given stategenerated behavioral field. The present behavioral field approach to general activity provides a methodology to investigate a variety of behavioral fields generated by chemical injections, brain stimulation, lesions, as well as behavioral preparations. Perhaps a thorough description of various state-generated behavioral fields is an important first step towards understanding learning processes, which modify not just a specific segment of behavior, but the whole behavioral field (Wong, 1977).

\section{REFERENCES}

ARCher, J. Tests for emotionality in rats and mice: A review. Animal Behaviour, 1973, 21, 205-235.

ARChER, J. Rodent sex differences in emotional and related behavior. Behavioral Biology, 1975, 14, 451-479.

Baumeister, A., Hawkins, W., \& Cromwell, R. Need states and activity level. Psychological Bulletin, 1964, 61, 438-453.

BerLyne, D. E. Curiosity and exploration: Animals spend much of their time seeking stimuli whose significance raises problems for psychology. Science, 1966, 153, 25-33.

BINDRA, D. The interrelated mechanisms of reinforcement and motivation, and the nature of their influence on response. In W. J. Arnold \& D. Levine (Eds.), Nebraska Symposium on Motivation. Lincoln, Nebr: University of Nebraska Press, 1969. Pp. 1-33.

Birke, L. I. A., \& ARChER, J. Open-field behavior of oestrous and dioestrous rats. Evidence against an 'emotionality' interpretation. Animal Behaviour, 1975, 23, 509-512.

Bolles, R. C. Grooming behavior in the rat. Journal of Comparative and Physiological Psychology, 1960, 53, 306-310.

Bolles, R. C. Theory of motivation. New York: Harper \& Row, 1967.
Bolles, R., \& Sanders, G. H. What does the ultrasonic activity recording device measure? Behavior Research Methods \& Instrumentation, 1969, 1, 180-183.

Brown, J. S. The motivation of behavior. New York: McGraw-Hill, 1961.

DeUTSCH, J. A. The structural basis of behavior. University of Chicago Press, 1960.

FINGER, F. W. Estrus and general activity in the rat. Journal of Comparative and Physiological Psychology, 1969, 68, 461-466.

GRAY, J. G. The psychology of fear and stress. New York: McGraw-Hill, 1971.

Gross, C. General activity. In L. Weiskrantz (Ed.), Analysis of behavioral change. New York: Harper \& Row, 1968.

HALLIDAY, M. S. Exploratory behavior. In L. Weiskrantz (Ed.), Analysis of behavioral change. New York: Harper \& Row, 1968.

Hughes, R. N. Effects of food deprivation, deprivation experience and sex on exploration in rats. British Journal of Psychology, $1968,59,47-53$.

Hull, C. L. Principles of behavior. New York: AppletonCentury-Crofts, 1943.

KING, J. A., \& WersmaN, R. G. Sanddigging contingent upon bar pressing in deermice (peromyscus). Animal Behaviour, $1964,12,446-450$.

Norton, S. Amphetamine as a model for hyperactivity in the rat. Physiology \& Behavior, 1973, 11, 181-186.

Russell, P. A. Sex differences in rats' stationary exploration as a function of stimulus and environmental novelty. Animal Learning \& Behavior, 1977, 5, 297-302.

SEGAL, E. F. Induction and the provenance of operants. In R. M. Gilbert \& J. R. Millenson (Eds.), Reinforcement: Behavioral analysis. New York: Academic Press, 1972. Pp. 1-34.

SHETTLEWORTH, S. J. Reinforcement and the organization of behavior in golden hamsters: Hunger, environment, and food reinforcement. Journal of Experimental Psychology: Animal Behavior Processes, 1975, 104, 56-87.

Valle, F. P. Motivation: Theories and issues. Monterey: Brooks/Cole, 1975.

VAlle, F. P., \& Bols, R. J. Age factors in sex differences in open-field activity of rats. Animal Learning \& Behavior, $1976,4,457.460$.

WANG, G. H. The relation between "spontaneous" activity and oestrus cycle in the white rat. Comparative Psychology Monograph, 1923, 2, No. 6.

Wovg, P. T. P. A behavioral field approach to instrumental learning in the rat: $I$. Partial reinforcement effects and sex differences. Animal Leaming \& Behavior, 1977, 5, 5-13.

Wong, P. T. P., RoAch, T., \& Osborne, B. A sand-digging apparatus for rats. Behavior Research Methods \& Instrumentation, 1975, 7, 34-36.

\section{NOTE}

1. Since the processing of Esterline Angus charts spanned 3 years and involved a succession of different research assistants, parts of the ultrasonic activity counts were misplaced and could not be located. However, on the basis of monitoring the ultrasonic data each day while the experiment was in progress, it was quite obvious that all females had higher ultrasonic counts than males, and no apparent systematic differences could be detected between the three deprivation conditions in females.

(Received for publication July 15, 1977; revision accepted May 4, 1978.) 